\title{
Fecal Lactoferrin and Other Stool Markers during Normal Pregnancy and in Inflammatory Bowel Diseases: A Prospective Study and Review of the Literature
}

\author{
James M. Gray ${ }^{\mathrm{a}}$ Kristin Knight ${ }^{\mathrm{a}}$ Vu Q. Nguyen ${ }^{\mathrm{a}}$ Marrieth G. Rubio ${ }^{\mathrm{a}}$ \\ Lauren Irby $^{b}$ James H. Boone ${ }^{b}$ Dario Sorrentino ${ }^{a, c}$ \\ a IBD Center, Division of Gastroenterology, Virginia Tech Carilion School of Medicine, Roanoke, VA, USA; \\ ${ }^{\mathrm{b}}$ TechLab Research and Development, Blacksburg, VA, USA; ${ }^{\mathrm{C} D e p a r t m e n t}$ of Clinical and Experimental Medical \\ Sciences, University of Udine School of Medicine, Udine, Italy
}

\section{Keywords}

Inflammatory markers - Pregnancy - Fecal lactoferrin . Intestinal mucosal inflammation - Inflammatory bowel diseases

\begin{abstract}
Introduction: Management of inflammatory bowel diseases (IBDs) - both Crohn's disease (CD) and ulcerative colitis (UC) - during pregnancy can be challenging since most monitoring tools available in nonpregnant patients are contraindicated. Objectives: The aim of the study was to test whether fecal inflammatory markers - specifically fecal lactoferrin physiologically change during normal pregnancy as a prerequisite to use them to monitor IBD activity during pregnancy. Methods: Fecal lactoferrin was tested in healthy pregnant and nonpregnant women from the same geographic area and age range (18-40 years) - all negative for clinical gastrointestinal tract inflammation. A retrospective review of fecal lactoferrin levels contrasted with the Simple Endoscopic Score for CD, and the Disease Activity Index for
\end{abstract}

karger@karger.com www.karger.com/iid

Karger"
(C) 2020 The Author(s)

Published by S. Karger AG, Basel

Karger

Open access

This article is licensed under the Creative Commons AttributionNonCommercial-NoDerivatives 4.0 International License (CC BYNC-ND) (http://www.karger.com/Services/OpenAccessLicense) Usage and distribution for commercial purposes as well as any distribution of modified material requires written permission.
UC was also performed in women with active IBDs within the same age range and geographical area. Results: In 30 nonpregnant subjects, fecal lactoferrin levels were $0.87 \pm 1.08$ $\mu \mathrm{g} / \mathrm{g}$. In 49 pregnant subjects, levels were $0.59 \pm 0.83,0.87 \pm$ 1.13 , and $0.85 \pm 1.06 \mu \mathrm{g} / \mathrm{g}$ during the first, second, and third trimester, respectively $(p=0.64)$, with average levels for the 3 trimesters of $0.81 \pm 1.04 \mu \mathrm{g} / \mathrm{g}$ ( $p=0.61$ compared to nonpregnant subjects). Sequential fecal lactoferrin levels $(n=26)$ did not differ from one trimester to the other in the individual subjects $(p=0.80$ ). In 45 female IBD patients ( 27 with CD and 18 with UC), fecal lactoferrin levels were correlated with disease activity as defined by the endoscopic scores: 218 , 688 , and $1,175 \mu \mathrm{g} / \mathrm{g}$ for CD and $931,2,088$, and $2,509 \mu \mathrm{g} / \mathrm{g}$ for UC, respectively, for mild, moderate, and severe activity. Conclusions: Fecal lactoferrin levels during normal pregnancy are superimposable to those of nonpregnant women and significantly below levels in women of the same childbearing age with active IBDs. Additional published data - reviewed in this atricle - and our own indicate that fecal lactoferrin and other markers can be potentially used to monitor disease activity in pregnant IBD patients.

(C) 2020 The Author(s)

Published by S. Karger AG, Basel

Dario Sorrentino

Division of Gastroenterology, Virginia Tech Carilion School of Medicine

3 Riverside Circle

Roanoke, VA 24016 (USA)

drsorrentino@ carilionclinic.org 


\section{Introduction}

Inflammatory bowel diseases (IBDs) - Crohn's disease (CD) and ulcerative colitis (UC) - are most often diagnosed in the second through fourth decades of life which, for female patients, coincide with their childbearing years $[1,2]$ when changes due to pregnancy may affect disease activity. Adverse outcomes such as gestational diabetes, preterm delivery, cesarean delivery, small for gestational age, stillbirth, spontaneous abortion, and congenital anomalies occur at an increased rate among women with active IBD [3-5]. Perhaps, due to the uncertainty of the disease impact on pregnancy, the rate of voluntary childlessness among women with IBDs is higher than that of the general population [6]. The rate of flares during pregnancy is related to disease activity at the time of conception. Increased disease activity at the time of conception is associated with an increased risk of flares later during the pregnancy [7]. Disease remission and flare prevention during pregnancy have been shown to decrease pregnancy-related complications [8]. Therefore, disease monitoring is crucial in this population. Endoscopy, the gold standard in IBD monitoring, carries significant risks during pregnancy. Both costly and invasive, endoscopy is usually reserved for emergency use during the second trimester [9]. It has been found to negatively impact the fetus and increase the risk for low gestational weight and preterm birth [10]. Imaging generally plays an important role in monitoring IBDs; however, in pregnant women, imaging might also be associated with risks. Computed tomography (CT) scans might pose risks of teratogenicity, and iodine-based contrast agents may negatively impact fetal thyroid function $[9,11]$. Magnetic resonance imaging (MRI) without contrast is considered safe during pregnancy - however, due to its scarce availability and cost, it is not always a viable monitoring technique for pregnant women. In addition, it has limited applications for colonic disease [12]. Small bowel ultrasound can detect inflammation, and it is safe during pregnancy, but its accuracy might not be enough to guide management, unless performed by expert operators [12]. Serum biomarkers used to assess inflammation and disease activity such as C-reactive protein (CRP), albumin, and hemoglobin have been found to be altered by normal pregnancy physiology and, therefore, may not correlate with disease activity [13]. Hence, the lack of simple, noninvasive tools to monitor the disease, detect a possible flare, and adjust therapy during pregnancy is a serious issue faced by pregnant women with IBDs.
Fecal biomarkers have emerged as an important tool in managing these diseases. They allow for noninvasive accurate monitoring of IBD activity without the risks associated with other methods. Among the others, fecal lactoferrin has been shown to be sensitive and specific for detecting active disease in IBD patients [14-16].

Lactoferrin, an $80-\mathrm{kDa}$ iron-binding glycoprotein, is expressed by activated neutrophils. Lactoferrin concentration in feces is the result of neutrophil translocation to the lining of the gastrointestinal tract during intestinal inflammation [17]. Therefore, the presence of lactoferrin in feces is indicative of an immune response and an indicator of inflammation. Scarce data currently exist for normal fecal lactoferrin levels in healthy pregnant women. In principle, like serum biomarkers, lactoferrin could be altered by normal pregnancy physiology and become inaccurate to monitor IBDs. Therefore, the aim of this study was to determine baseline levels of lactoferrin during pregnancy in healthy women.

\section{Materials and Methods}

In this study, 3 distinct subject populations were studied. Group 1 was composed of healthy nonpregnant women of childbearing age from southwest Virginia, USA, and served as controls. The stool samples were collected during an open sample drive performed by TechLab (Blacksburg, VA, USA). Fecal samples were collected at home by the subjects using standard methods. Individuals in this group (1) were between the ages of 18 and 40 years; (2) had no history of IBDs or other known upper or lower gastrointestinal tract diseases (including but not limited to celiac disease, irritable bowel syndrome, diverticulitis, and Clostridium difficile colitis); and (3) were not using NSAIDs or any other potentially toxic medications for the gastrointestinal tract. Subjects who had been diagnosed with an immune-mediated inflammatory disease(s) that could potentially be associated with inflammation of the intestine were also excluded.

Group 2 was composed of healthy pregnant women from the same geographical area of the controls who provided fecal samples throughout their pregnancy during each of the 3 trimesters to be tested for fecal lactoferrin. A maximum of 3 samples, one from each trimester, was collected from each subject. These fecal samples were collected from the patient population of Carilion Clinic's Obstetrics \& Gynecology Department and local private practices outside Carilion Medical Center. These subjects at the time of sample collection had a normal uncomplicated pregnancy. Eligibility criteria for group 2 were identical to those of group 1 with the addition of a positive pregnancy status confirmed by a physician. Subjects who were seeing physicians outside of the Carilion Clinic network were asked to provide their medical history from their physician's office. For the others, the medical history was available in their electronic medical records. Fecal samples were collected at home by the subjects using standard methods and brought with them during their scheduled appointments with the obstetrician 
Table 1. Healthy pregnant subjects characteristics $(n=49)$

\begin{tabular}{lc}
\hline Demographics & \\
Age in years, range & $18-40$ \\
Ethnicity & $31(63)$ \\
White, non-Hispanic, $n(\%)$ & $14(29)$ \\
Black, $n(\%)$ & $3(6)$ \\
Hispanic, $n(\%)$ & $1(2)$ \\
Biracial, $n(\%)$ & \\
Social history & $16(33), 9(18)$, and \\
Tobacco use: current, former, and & $24(49)$ \\
never, $n(\%)$ & $4(8), 5(10)$, and \\
Marijuana use: current, former, and & $40(82)$ \\
never, $n(\%)$ &
\end{tabular}

or at a set time and location. Participating individuals received reimbursement for their time. For each subject, the demographics, medical history (including social history), and clinical parameters were collected during the study. Laboratory studies in addition to fecal lactoferrin included standard routine blood tests. All fecal samples were kept at $-20^{\circ} \mathrm{C}$ until the time of transportation to the lab for analysis. Tests were performed at TechLab, Blacksburg, VA. Fecal lactoferrin was measured by enzyme immunoassay, and results are reported as $\mu \mathrm{g} / \mathrm{g}$ feces. Group 3 was composed of nonpregnant women with active IBDs (UC or CD). This group was used as a reference group to verify correlation of fecal lactoferrin with disease activity in this population of childbearing age (18-40 years) all coming from the same geographical area of the subjects of group 1 and 2 . The subjects were identified utilizing a retrospective chart review of previously established IBD patients with Carilion Clinic Medical Center. Inclusion criteria for this group included (1) clinically active IBDs with recent endoscopy and reported Simple Endoscopic Score for CD (SES-CD) or the endoscopic component of the Mayo Clinical Score (Disease Activity Index [DAI]) for UC [18], (2) fecal lactoferrin tested within 30 days of endoscopy, and (3) verified nonpregnant status. The SESCD is defined as follows: remission (score $0-2$ ), mild inflammation (score 3-6), moderate inflammation (score 7-15), and severe inflammation (score $\geq 16$ ). The endoscopic component of the DAI is defined as follows: normal or inactive disease (score 0 ), mild disease (score 1), moderate disease (score 2), and severe disease (score 3).

The study protocol was approved by the Carilion Clinic Institutional Review Board. Subjects who agreed to participate signed the informed consent.

\section{Results}

Group 1 - nonpregnant healthy women within 18-40 years of age - included 30 subjects tested for fecal lactoferrin levels. Levels ranged from 0.01 to $3.81 \mu \mathrm{g} / \mathrm{g}$ with a mean level \pm SD of $0.87 \pm 1.08 \mu \mathrm{g} / \mathrm{g}$.

Fecal Biomarkers in Pregnancy and Inflammatory Bowel Diseases
Table 2. IBD patients characteristics $(n=45)$

$\begin{array}{lr}\text { Demographics } & \\ \text { Age in years, range } & 18-40 \\ \text { Ethnicity } & \\ \quad \text { White, non-Hispanic, } n(\%) & 40(89) \\ \text { Black, } n(\%) & 5(11) \\ \text { Social history } & 4(9), 18(40) \text {, and } \\ \text { Tobacco use: current, former, and } & 23(51) \\ \quad \text { never, } n(\%) & \\ \text { Disease characteristics } & \\ \text { Disease type } & 27(60) \\ \text { CD, } n(\%) & 18(40) \\ \text { UC, } n(\%) & 3.7(5.3) \\ \text { Disease duration in years (SD) } & \\ \text { SES-CD } & 8(30) \\ \text { Mild, } n(\%) & 8(30) \\ \text { Moderate, } n(\%) & 11(40) \\ \text { Severe, } n(\%) & \\ \text { DAI (UC) } & 6(33) \\ \text { Mild, } n(\%) & 5(28) \\ \text { Moderate, } n(\%) & 7(39) \\ \text { Severe, } n(\%) & \end{array}$

IBD, inflammatory bowel disease; CD, Crohn's disease; UC, ulcerative colitis; SES-CD, Simple Endoscopic Score for CD; DAI, Disease Activity Index.

Group 2 - healthy pregnant women - included 49 subjects within 18-40 years of age. Their clinical features are shown in Table 1. A minority of these subjects were tobacco or marijuana users. Mean levels were obtained by pooling the results of all enrolled study subjects per trimester. During the first trimester, mean fecal lactoferrin levels were $0.59 \pm 0.83 \mu \mathrm{g} / \mathrm{g}$; during the second trimester, the mean lactoferrin levels were $0.87 \pm 1.13 \mu \mathrm{g} / \mathrm{g}$; and during the third trimester, the levels were $0.85 \pm 1.06 \mu \mathrm{g} / \mathrm{g}$. The mean fecal lactoferrin value for pregnant women over all 3 trimesters $(0.81 \pm 1.04 \mu \mathrm{g} / \mathrm{g})$ was not different from the mean levels in healthy women of group $1(p=$ 0.61 ). There was also no difference between the mean fecal lactoferrin values between the 3 trimesters (ANOVA test: $p=0.64)$. Sequential individual samples were available for 26 subjects. Their fecal lactoferrin values did not differ from 1 trimester to the other in the individual subjects (ANOVA $p=0.80$ ). Two of the study subjects in this group had elevated fecal lactoferrin levels. One of them had a value of $86 \mu \mathrm{g} / \mathrm{g}$ during the third trimester with normal levels during the first and second trimesters ( 0.39 and $0.78 \mu \mathrm{g} / \mathrm{g}$, respectively). This subject's pregnancy was complicated by excessive maternal weight gain (with a BMI of 54), maternal depression, gestational hyperten-

Inflamm Intest Dis 2020;5:151-157 153 


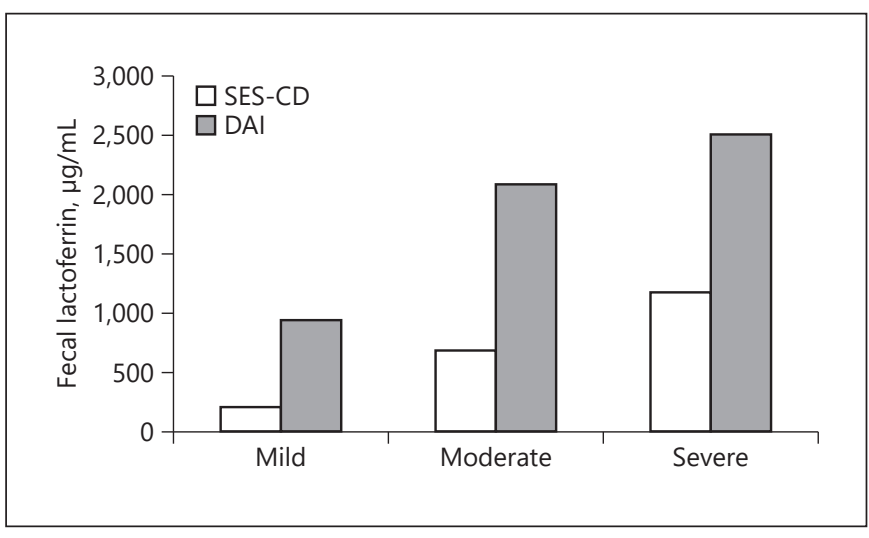

Fig. 1. Fecal lactoferrin values in relation to endoscopy scores (SES-CD for CD patients and DAI for UC patients) in a group of female IBD patients within the same age range (18-40 years) of the pregnant subjects. Values were correlated with disease activity. IBD, inflammatory bowel disease; CD, Crohn's disease; UC, ulcerative colitis; SES-CD, Simple Endoscopic Score for CD; DAI, Disease Activity Index.

sion, and tobacco, marijuana, and alcohol abuse. Additionally, this subject had an increased WBC count (17.7 $\mathrm{K} / \mu \mathrm{L}$ ) during the third trimester. She experienced arrest of dilation during labor and delivered the fetus by cesarean section. At time of delivery, the infant appeared healthy. Due to the presence of confounding factors and possible risks for gastrointestinal tract inflammation, she was excluded from the analyses. The second patient had elevated fecal lactoferrin levels during the first trimester $(28 \mu \mathrm{g} / \mathrm{g})$. The subject did not collect samples during the second or third trimester having started NSAIDs. She had a complicated pregnancy exacerbated by uncontrolled type 2 diabetes, hyperlipidemia, multiple urinary tract infections, and gestational hypertension. She also had a history of complicated pregnancies with fetal anomalies, preterm deliveries, and stillbirths. At the time of delivery, no abnormalities or medical issues for the mother or infant were recorded. Again, due to the presence of confounding factors and a complicated pregnancy, the data relative to this patient were excluded from the analyses.

Group 3 - nonpregnant women with active IBDs - included 45 subjects within 18-40 years of age of whom 27 had CD and 18 UC (Table 2). The distribution of SES-CD and DAI scores for mild, moderate, and severe disease was similar in this group. Figure 1 shows the lactoferrin values - performed within 30 days of endoscopy - as they relate to the endoscopy scores in this group of female patients. For CD patients, the mean lactoferrin levels for mild, moderate, and severe disease as defined by the SES-
CD score were 217,688 , and $1,175 \mu \mathrm{g} / \mathrm{g}$, respectively. For UC patients, mean lactoferrin levels for mild, moderate, and severe disease as defined by the DAI score were 931, 2,088 , and $2,509 \mu \mathrm{g} / \mathrm{g}$, respectively.

\section{Discussion}

Pregnancy poses challenges for women with IBDs. Many feel uncertain about treatment during pregnancy - mainly because of potential side effects of the medications on their unborn child [6]. Indeed, many of the medications used to treat IBDs have been found in the breast milk of nursing mothers $[19,20]$. Fears of heritability, risk of congenital abnormalities, and medication teratogenicity all effect the decision to bear children for women with IBDs.

Monitoring disease activity and diagnosing a flare poses special problems during pregnancy since typical tools such as endoscopy and imaging are contraindicated except in emergency circumstances. Endoscopy might be associated with an increased risk of complications such as low gestational weight and preterm birth [10]. Imaging - including CT, MRI, and ultrasound - also has limitations and shortcomings. CT increases the risk of teratogenicity and is contraindicated during pregnancy. Noncontrast MRI is considered safe, but cost and availability are a major limiting factor in addition to its limited accuracy for colonic disease [12]. Intestinal ultrasound is safe even during pregnancy; however, it is an imaging test that requires special expertise and it is not widely available [12].

Fecal biomarkers, such as lactoferrin, are accurate indicators of inflammation as well as being noninvasive and inexpensive [21-25]. Hence, they could be ideally suited to monitor IBD activity during pregnancy. However, pregnancy is known to be associated with a number of physiological changes such as systemic hormonal shifts and immunological changes [26-28] which might be partly responsible for several alterations in hematological parameters, including leukocyte number [29]. Hence, it is important to verify that inflammatory markers do not change during normal pregnancy as a prerequisite to their use to monitor disease activity in pregnant IBD patients. In this study, we focused on fecal lactoferrin which appears to be an accurate predictor of IBD flares in nonpregnant patients $[15,16,24]$. We compared fecal lactoferrin values during normal pregnancy to those of healthy nonpregnant women and to those of women with active IBDs of similar age and geographical provenance. 
Our results show that fecal lactoferrin levels do not change during any of the 3 trimesters of pregnancy compared to those of nonpregnant controls. We did observe elevated biomarker levels in 2 women with a significant medical history and complicated pregnancies. Due to the lack of additional information related to the gastrointestinal tract in these 2 subjects, the mechanism(s) at the basis of their increased fecal lactoferrin levels was unclear. We cannot exclude intestinal ischemia as a consequence of a number of comorbidities or a preexisting but asymptomatic gastrointestinal disease or an undetected infection all of which could lead to intestinal inflammation and release of lactoferrin [30,31]. Since by definition, these subjects did not have a normal pregnancy, they were excluded from data analyses. Fecal lactoferrin levels appeared stable and below the threshold of normality in all the other subjects during the 3 trimesters assessed as a group or sequentially in individual subjects. In this study, we also confirmed that fecal lactoferrin is an accurate marker of IBD activity in this population of childbearing age. In 45 nonpregnant patients with active IBDs within 18-40 years of age, we showed that the marker levels assessed at the time of colonoscopy closely correlated with the disease activity as determined by the endoscopic score in both UC and CD patients. The limitations of this study are the relatively small sample size and the lack of detailed characterization of the enrolled subjects who were considered to be healthy only on clinical grounds. Our data corroborate a number of other studies related to the use of fecal biomarkers in normal pregnancy and as a monitoring tool for IBDs [32, 33].

A number of studies have focused on fecal marker levels during normal pregnancy [34-36] and in pregnant women with IBDs. In the study by Barré et al. [34], the authors found that there were no differences in fecal lactoferrin levels between any trimester and prepregnancy levels $(1.52 \mu \mathrm{g} / \mathrm{g}$ during pregnancy vs. $1.08 \mu \mathrm{g} / \mathrm{g}$ prepregnancy $[p=0.08])$ and, therefore, concluded that pregnancy has no effect on fecal lactoferrin levels. Likewise, there were no differences between prepregnancy and pregnancy levels in IBD patients. However, fecal lactoferrin was significantly higher in IBD patients compared to healthy pregnant controls at each trimester [34]. Similar results in a small group of patients have been published in abstract form by Koslowsky et al. [35] and, earlier, with fecal calprotectin by Julsgaard [36]. Bálint et al. [37] also did not find significant differences in fecal calprotectin concentrations between pregnant and nonpregnant healthy women.

Fecal Biomarkers in Pregnancy and Inflammatory Bowel Diseases
Other studies have focused on the correlation of fecal biomarker levels with disease activity in IBDs as assessed by clinical activity indices. Rottenstreich et al. [38] studied in 157 pregnancies the correlation between fecal calprotectin levels in IBD patients during preconception, first trimester, second trimester, third trimester, and postpartum. Higher fecal calprotectin levels were found in all subjects who had active disease with fecal calprotectin levels significantly correlated with physician global assessment (PGA) and disease activity indices in all 5 periods. Furthermore, fecal calprotectin was noted to be significantly higher in those patients who experienced a disease flare later in the gestation period compared to those who maintained clinical remission [38]. Barré et al. [34] found that during the third trimester, fecal lactoferrin levels significantly correlated with PGA, modified Harvey-Bradshaw Index (HBI) for CD, and partial Mayo Score for UC. Likewise, Huang et al. [39] reported that women with IBDs who had clinically active disease during preconception and pregnancy had higher fecal calprotectin levels than women who had clinically inactive disease.

Kammerlander et al. [40] studied 219 pregnant women with moderate-severe IBDs (as assessed by HBI/patient-based Simple Clinical Colitis Activity Index or physician assessment) in treatment with anti-TNF agents and showed fecal calprotectin levels to be significantly higher in active compared to quiescent disease with a correlation of the biomarker level to disease activity in all gestational periods. They also found a fecal calprotectin sensitivity of $69.7-80.0 \%$, a specificity of $66.7-73.3 \%$, and a positive predictive value of $66.7-74.4 \%$ over the 4 gestational periods (preconception and the 3 trimesters) when a cutoff of $200 \mathrm{mg} / \mathrm{kg}$ was used. They found no clinically significant differences in CRP, albumin, or hemoglobin [40]. In another study, Julsgaard et al. [36] measured fecal calprotectin levels in healthy pregnant women and compared them to those of pregnant women with active IBDs as assessed by HBI/Simple Clinical Colitis Activity Index or PGA. Patients with active disease showed levels higher than controls, and the authors concluded that fecal calprotectin is a good indicator of IBD activity in pregnancy [36].

In another study, Kanis et al. [41] showed that fecal calprotectin shows an overall high sensitivity and specificity to identify disease activity. However, the usefulness of the biomarker to predict a clinical relapse at the subsequent trimester or postpartum was considered poor. In that study, the authors considered fecal calprotectin values $>200 \mu \mathrm{g} / \mathrm{g}$ as abnormal. However, they did not estab- 
lish the individual threshold value above which a clinical flare can be expected. Such value might considerably differ among patients based upon their baseline disease burden.

It has been rightly pointed out that there is a lack of studies comparing the accuracy of stool markers against the IBD gold-standard method (endoscopy) for assessing disease activity during pregnancy [32,33]. Clinical scores, which have been used to assess the markers' accuracy, mostly reflect subjective symptoms which are difficult to assess during pregnancy. Regardless, both fecal lactoferrin and calprotectin appear superior to any other noninvasive marker (such as CRP, hemoglobin, and albumin) to monitor IBDs during pregnancy [32].

In conclusion, our study supports other observations and shows that fecal lactoferrin levels during normal pregnancy are superimposable to those of nonpregnant women and significantly below levels of nonpregnant women of the same childbearing age with active IBDs. Current data indicate that stool marker levels (both lactoferrin and calprotectin) correlate to disease activity during pregnancy. Hence, they can be potentially used to monitor disease activity in pregnant IBD patients.

\section{Acknowledgements}

The authors would like to thank Dr. Allison Durica, Dr. Megan Lord, Dr. Emily Evans-Hoeker and the nursing staff at Carilion's Obstetrics and Gynecology, Maternal and Fetal Medicine, and Reproductive Medicine and Fertility Clinics for their assistance in recruitment and sample collection.

\section{Statement of Ethics}

All research performed in this study was in accordance with the ethical standards set forth by the Institutional Review Board of Carilion Clinic and federal regulations. Written informed consent was obtained from all patients for the use of data for analysis and publication.

\section{Conflict of Interest Statement}

D.S. has received consulting fees from Abbott/AbbVie, Schering-Plough, MSD, Janssen Research \& Development, LLC., Centocor Inc., TechLab, Hoffmann-LaRoche, Giuliani, ScheringPlough, and Ferring; research grants from AbbVie, Janssen Research \& Development, LLC, Schering-Plough, TechLab, Centocor, and serves in the Speakers Bureau of AbbVie and the National Faculty of Janssen. V.Q.N. has received grant support from AbbVie Inc. M.G.R. has received grant support from Gilead. L.I. and J.B. are currently employees of TechLab Inc., Blacksburg VA, USA. The other authors have no conflicts to declare.

\section{Funding Sources}

The study was funded by an unrestricted research grant from TechLab, Blacksburg VA, USA.

\section{Author Contributions}

D.S. and J.H.B. designed the study; J.M.G., D.S., and V.Q.N. conceived the manuscript; J.M.G. and K.K. identified, consented, and collected samples from patients; J.H.B. and L.I. performed laboratory procedures. J.M.G., M.G.R., and D.S. collected all clinical data. All authors reviewed the manuscript and approved the final draft.

\section{References}

1 Damas OM, Deshpande AR, Avalos DJ, Abreu MT. Treating inflammatory bowel disease in pregnancy: the issues we face today. J Crohns Colitis. 2015 Oct;9(10):928-36.

2 Bar-Gil Shitrit A, Grisaru-Granovsky S, Ben Ya'acov A, Goldin E. Management of inflammatory bowel disease during pregnancy. Dig Dis Sci. 2016 Aug;61(8):2194-204.

3 Boyd HA, Basit S, Harpsøe MC, Wohlfahrt J, Jess T. Inflammatory bowel disease and risk of adverse pregnancy outcomes. PLoS One. 2015;10(6):e0129567.

4 Bröms G, Granath F, Linder M, Stephansson O, Elmberg M, Kieler H. Birth outcomes in women with inflammatory bowel disease: effects of disease activity and drug exposure. Inflamm Bowel Dis. 2014 Jun;20(6):1091-8.
5 Cornish J, Tan E, Teare J, Teoh TG, Rai R, Clark SK, et al. A meta-analysis on the influence of inflammatory bowel disease on pregnancy. Gut. 2007 Jun;56(6):830-7.

6 Mountifield R, Bampton P, Prosser R, Muller $\mathrm{K}$, Andrews JM. Fear and fertility in inflammatory bowel disease: a mismatch of perception and reality affects family planning decisions. Inflamm Bowel Dis. 2009 May; 15(5): $720-5$.

7 Abhyankar A, Ham M, Moss AC. Meta-analysis: the impact of disease activity at conception on disease activity during pregnancy in patients with inflammatory bowel disease. Aliment Pharmacol Ther. 2013 Sep;38(5): $460-6$.
8 Pinder M, Lummis K, Selinger CP. Managing inflammatory bowel disease in pregnancy: current perspectives. Clin Exp Gastroenterol. 2016;9:325-35.

9 Tran TT, Ahn J, Reau NS. ACG clinical guideline: liver disease and pregnancy. Am J Gastroenterol. 2016 Feb;111(2):176-96.

10 Ludvigsson JF, Lebwohl B, Ekbom A, Kiran RP, Green PH, Höijer J, et al. Outcomes of pregnancies for women undergoing endoscopy while they were pregnant: a nationwide cohort study. Gastroenterology. 2017 Feb; 152(3):554-e9.

11 Murji A, Crosier R, Rasuli P. Non-obstetric diagnostic imaging in pregnancy. CMAJ. 2015 Nov 17;187(17):1309. 
12 Benevento G, Avellini C, Terrosu G, Geraci M, Lodolo I, Sorrentino D. Diagnosis and assessment of Crohn's disease: the present and the future. Expert Rev Gastroenterol Hepatol. 2010 Dec;4(6):757-66.

13 Klajnbard A, Szecsi PB, Colov NP, Andersen MR, Jørgensen M, Bjørngaard B, et al. Laboratory reference intervals during pregnancy, delivery and the early postpartum period. Clin Chem Lab Med. 2010 Feb;48(2):237-48.

14 Dai C, Jiang M, Sun MJ. Fecal markers in the management of inflammatory bowel disease. Postgrad Med. 2018 Sep;130(7):597-606.

15 Dai C, Jiang M, Sun MJ, Cao Q. Fecal lactoferrin for assessment of inflammatory bowel disease activity: a systematic review and metaanalysis. J Clin Gastroenterol. 2020 Jul;54(6): 545-53.

16 Dai J, Liu WZ, Zhao YP, Hu YB, Ge ZZ. Relationship between fecal lactoferrin and inflammatory bowel disease. Scand J Gastroenterol. 2007;42(12):1440-4

17 Actor JK, Hwang SA, Kruzel ML. Lactoferrin as a natural immune modulator. Curr Pharm Des. 2009;15(17):1956-73.

18 D'Haens G, Sandborn WJ, Feagan BG, Geboes $\mathrm{K}$, Hanauer SB, Irvine EJ, et al. A review of activity indices and efficacy end points for clinical trials of medical therapy in adults with ulcerative colitis. Gastroenterology. 2007; 132(2):763-86

19 Shannahan SE, Erlich JM, Peppercorn MA Insights into the treatment of inflammatory bowel disease in pregnancy. Therap Adv Gastroenterol. 2019;12:1756284819852231.

20 Johns DG, Rutherford LD, Leighton PC, Vogel CL. Secretion of methotrexate into human milk. Am J Obstet Gynecol. 1972 Apr 1; 112(7):978-80

21 Soubières AA, Poullis A. Emerging role of novel biomarkers in the diagnosis of inflammatory bowel disease. World J Gastrointest Pharmacol Ther. 2016 Feb 6;7(1):41-50.

22 Knyazev OV, Kagramanova AV, Korneeva IA, Noskova KK, Belousov SV, Parfenov AI. The use of fecal calprotectin in monitoring activity of inflammatory bowel diseases. Ter Arkh. 2019 May 16;91(4):53-61.
23 Siddiqui I, Majid H, Abid S. Update on clini$\mathrm{cal}$ and research application of fecal biomarkers for gastrointestinal diseases. World J Gastrointest Pharmacol Ther. 2017 Feb 6;8(1): 39-46.

24 Rubio MG, Amo-Mensah K, Gray JM, Nguyen VQ, Nakat S, Grider D, et al. Fecal lactoferrin accurately reflects mucosal inflammation in inflammatory bowel disease. World J Gastrointest Pathophysiol. 2019 Dec 31;10(5): 54-63.

25 Langhorst J, Elsenbruch S, Mueller T, Rueffer A, Spahn G, Michalsen A, et al. Comparison of 4 neutrophil-derived proteins in feces as indicators of disease activity in ulcerative colitis. Inflamm Bowel Dis. 2005 Dec;11(12):108591.

26 Soma-Pillay P, Nelson-Piercy C, Tolppanen $\mathrm{H}$, Mebazaa A. Physiological changes in pregnancy. Cardiovasc J Afr. 2016 Mar-Apr;27(2): 89-94.

27 Mor G, Cardenas I. The immune system in pregnancy: a unique complexity. Am J Reprod Immunol. 2010 Jun;63(6):425-33.

28 Robinson DP, Klein SL. Pregnancy and pregnancy-associated hormones alter immune responses and disease pathogenesis. Horm Behav. 2012 Aug;62(3):263-71.

29 Chandra S, Tripathi AK, Mishra S, Amzarul $M$, Vaish AK. Physiological changes in hematological parameters during pregnancy. Indian J Hematol Blood Transfus. 2012 Sep;28(3): 144-6.

30 Pope MR, Fleming SD. TLR2 modulates antibodies required for intestinal ischemia/reperfusion-induced damage and inflammation. J Immunol. 2015 Feb 1;194(3):1190-8.

31 Swale A, Miyajima F, Roberts P, Hall A, Little $\mathrm{M}$, Beadsworth MB, et al. Calprotectin and lactoferrin faecal levels in patients with Clostridium difficile infection (CDI): a prospective cohort study. PLoS One. 2014;9(8): e106118.

32 Tandon P, Leung K, Yusuf A, Huang VW. Noninvasive methods for assessing inflammatory bowel disease activity in pregnancy: a systematic review. J Clin Gastroenterol. 2019 Sep;53(8):574-81.
33 Choden T, Mandaliya R, Charabaty A, Mattar MC. Monitoring inflammatory bowel disease during pregnancy: current literature and future challenges. World J Gastrointest Pharmacol Ther. 2018;9(1):1-7.

34 Barré A, Tarassishin L, Eisele C, Hu J, Nair N, Stone J, et al. P160 Faecal Lactoferrin is a reliable IBD biomarker during pregnancy. Crohns Colitis. 2018 Feb;12(Suppl 1):S178.

35 Koslowsky B, Livovsky DM, Goldin E, BarGil Shitrit A. P307 fecal lactoferrin levels are stable during pregnancy. J Crohns Colitis. 2017 Feb;11(Suppl 1):S233.

36 Julsgaard M, Hvas CL, Gearry RB, Vestergaard T, Fallingborg J, Svenningsen L, et al. Fecal calprotectin is not affected by pregnancy: clinical implications for the management of pregnant patients with inflammatory bowel disease. Inflamm Bowel Dis. 2017 Jul;23(7): 1240-6.

37 Bálint A, Berényi A, Farkas K, É Pallagi Kunstár, Altorjay Â, Csonka A, et al. Pregnancy does not affect fecal calprotectin concentration in healthy women. Turk J Gastroenterol. 2017 May;28(3):171-5.

38 Rottenstreich A, Mishael T, Granovsky SG, Koslowsky B, Schweistein H, Abitbol G, et al. Clinical utility of fecal calprotectin in monitoring disease activity and predicting relapse in pregnant patients with inflammatory bowel diseases. Eur J Intern Med. 2020 Mar 17; S0953-6205(20):30111-4.

39 Huang V, Bal J, Foshaug RR, Ambrosio L, Kroeker K, Dieleman LA, et al. Su1255 fecal calprotectin is elevated with clinical disease activity during pregnancy in women with inflammatory bowel disease. Gastroenterology. 2015; April;148(4):S-452.

40 Kammerlander $\mathrm{H}$, Nielsen J, Kjeldsen J, Knudsen T, Gradel KO, Friedman S, et al. Fecal calprotectin during pregnancy in women with moderate-severe inflammatory bowel disease. Inflamm Bowel Dis. 2018 Mar 19; 24(4):839-48.

41 Kanis SL, de Lima A, van der Ent C, Van Der Woude CJ. Sa1941 anti-TNF cord levels are significantly higher after IFX exposure during pregnancy compared to ADA exposure. Gastroenterology. 2016;April;150(4):S410. 